\title{
Role of passive T-cell death in chronic experimental autoimmune encephalomyelitis
}

\author{
Shohreh Issazadeh, ${ }^{1}$ Kald Abdallah, ${ }^{2}$ Tanuja Chitnis, ${ }^{1}$ Anil Chandraker, ${ }^{2}$ \\ Andrew D. Wells, ${ }^{3}$ Laurence A. Turka, ${ }^{3}$ Mohamed H. Sayegh, ${ }^{2}$ and Samia J. Khoury ${ }^{1}$ \\ ${ }^{1}$ Center for Neurologic Diseases, and \\ ${ }^{2}$ Laboratory of Immunogenetics and Transplantation, Brigham and Women's Hospital, Harvard Medical School, \\ Boston, Massachusetts, USA \\ ${ }^{3}$ Department of Medicine, University of Pennsylvania, Philadelphia, Pennsylvania, USA \\ Address correspondence to: Samia J. Khoury, Center for Neurologic Diseases, 77 Avenue Louis Pasteur, Boston, \\ Massachusetts 02115, USA. Phone: (617) 525-5370; Fax: (617) 525-5252; E-mail: khoury@cnd.bwh.harvard.edu.
}

Shohreh Issazadeh's present address is: Medical Inflammation Research, Cell \& Molecular Biology,

University of Lund, Lund, Sweden.

Shohreh Issazadeh, Kald Abdallah, and Tanuja Chitnis contributed equally to this work.

Received for publication October 4, 1999, and accepted in revised form March 7, 2000.

\begin{abstract}
The mechanisms of chronic disease and recovery from relapses in experimental autoimmune encephalomyelitis (EAE), an animal model of multiple sclerosis, are unknown. Deletion of myelin-specific lymphocytes by apoptosis may play a role in termination of the inflammatory response. One pathway of apoptosis is the passive cell death or "cell death by neglect" pathway, which is under the control of the Bcl family of genes. To investigate the role of passive cell death pathway in EAE, we used mice with transgenic expression of the long form of the $b c l-x$ gene $\left(\mathrm{Bcl}-\mathrm{x}_{\mathrm{L}}\right)$ targeted to the T-cell lineage. We found that mice transgenic for $\mathrm{Bcl}-\mathrm{x}_{\mathrm{L}}$ have an earlier onset and a more chronic form of EAE induced by myelin oligodendrocyte glycoprotein (MOG) peptide 35-55 compared with wild-type littermate mice. This was not due to an expanded autoreactive cell repertoire. Primed peripheral lymphocytes from Bcl- $\mathrm{x}_{\mathrm{L}}$ transgenic mice showed increased proliferation and cytokine production to MOG peptide in vitro compared with lymphocytes from wild-type animals. Immunohistologic studies demonstrated increased cellular infiltrates, immunoglobulin precipitation, and demyelination in the Bcl- $\mathrm{x}_{\mathrm{L}}$ transgenic central nervous system (CNS) compared with controls. There was also a decreased number of apoptotic cells in the CNS of Bcl- $\mathrm{x}_{\mathrm{L}}$ transgenic mice when compared with littermates at all time points tested. This is the first report of an autoimmune disease model in $\mathrm{Bcl}-\mathrm{x}_{\mathrm{L}}$ transgenic mice. Our data indicate that the passive cell death pathway is important in the pathogenesis of chronic EAE. These findings have implications for understanding the pathogenesis of multiple sclerosis and other autoimmune diseases.
\end{abstract}

J. Clin. Invest. 105:1109-1116 (2000).

\section{Introduction}

In experimental autoimmune encephalomyelitis (EAE), myelin-specific $\mathrm{T}$ lymphocytes recognize and are activated by central nervous system (CNS) myelin antigens. Recruitment of antigen nonspecific cells follows and perpetrates the local inflammatory response. Several regulatory mechanisms function to terminate responses to antigens; these include cytokine regulation, apoptosis through activation-induced cell death (AICD), and apoptosis resulting from cytokine withdrawal (1). Deletion of activated lymphocytes by apoptosis in the CNS may play a role in termination of the inflammatory response in EAE, and coincide with clinical recovery (2).

Two distinct pathways leading to T-cell apoptosis have been described: passive cell death and AICD. Passive cell death is mediated by growth factor deprivation of activated T cells (3-6) and can be accomplished experimentally by blocking the pathways of IL-2 production (such as by costimulatory signal blockade) (7). In contrast, AICD occurs when primed $\mathrm{T}$ cells are repeatedly exposed to antigen, and it is mediated by Fas/Fas ligand (FasL) interaction $(8,9)$ and is dependent on previous exposure to IL-2 (10).

The role of the Fas/FasL pathway in EAE was investigated in mice expressing lpr (Fas) and gld (FasL) mutations. These mice were found to be resistant to the development of clinical EAE, and fewer apoptotic cells were detected in inflammatory lesions of lpr mice than in wild-type lesions of similar severity. The relative resistance to EAE was attributed to the failure of oligodendrocyte apoptosis $(11,12)$. The role of Fas/FasL remains controversial; however, recent work by Suvannavejh et al. (13) in Fas-deficient SJL mice suggests that Fas-mediated apoptosis plays an important role in regulation of autoimmune responses.

To study the role of passive cell death in EAE, we used mice with transgenic expression of the long from of the $b c l-x$ gene $\left(\mathrm{Bcl}-\mathrm{x}_{\mathrm{L}}\right)$ targeted to the T-cell lineage $(3,4)$. In normal mice, $\mathrm{Bcl}-\mathrm{x}_{\mathrm{L}}$ is minimally expressed in resting $\mathrm{T}$ cells, but is induced by TCR ligation, and this induction 
is augmented and prolonged by CD28 costimulation (4, 14-16). In Bcl- $\mathrm{x}_{\mathrm{L}}$ transgenic mice, $\mathrm{Bcl}-\mathrm{x}_{\mathrm{L}}$ expression is detectable in unstimulated $\mathrm{T}$ cells and is constitutively expressed after activation of $\mathrm{T}$ cells with or without $\mathrm{CD} 28$ costimulation (3). Thymocytes from Bcl- $\mathrm{x}_{\mathrm{L}}$ transgenic mice are resistant to apoptosis induced by glucocorticoids, $\gamma$-irradiation, calcium ionophore, and CD3 cross-linking, but they are not protected from clonal deletion by self-antigens (3). We have previously found that transgenic expression of $\mathrm{Bcl}-\mathrm{x}_{\mathrm{L}}$ does not obviate the requirement for $\mathrm{CD} 28$ signaling in T-cell proliferation, and although $\mathrm{Bcl}-\mathrm{x}_{\mathrm{L}}$ expression protects $\mathrm{T}$ cells from apoptosis resulting from growth factor insufficiency, they were still sensitive to AICD (17).

In this report, we found that $\mathrm{Bcl}-\mathrm{x}_{\mathrm{L}}$ transgenic mice immunized with myelin oligodendrocyte glycoprotein peptide 35-55 (MOG p35-55) developed more severe EAE than nontransgenic littermates. This was associated with increased antigen-specific proliferation, cytokine production by primed $\mathrm{T}$ cells, and more severe pathology in the CNS. More importantly, the Bcl- $\mathrm{x}_{\mathrm{L}}$ transgenic mice had fewer apoptotic cells in the CNS compared with littermates at all time points tested. These data underscore the importance of T-cell apoptosis in terminating the inflammatory response in EAE and suggest that failure to terminate T-cell responses in the CNS may be a mechanism for the development of chronic disease.

\section{Methods}

Mice. Bcl- $\mathrm{x}_{\mathrm{L}}$ transgenic mice originally provided by $\mathrm{G}$. Nunez (University of Michigan, Ann Arbor, Michigan, USA) were back-crossed onto a B6 background for 9 generations. The animals were bred in our facility and were screened for the presence of the transgene by PCR. The primers were forward: GCGGGCATTCAGTGACCTGA, and reverse: TAAGTGGCCATCCAAGCTGC. Sex and age-matched wild-type littermates were used as controls (17).

EAE-induction with MOG peptide. MOG p35-55 (M-EV-G-W-Y-R-S-P-F-S-R-O-V-H-L-Y-R-N-G-K) corresponding to mouse sequence was synthesized by Quality
Controlled Biochemicals Inc. (Hopkinton, Massachusetts, USA) and purified by HPLC. Peptide purity was greater than 99\% after HPLC. Experiments were performed with 3-8 animals in each group. Bcl- $x_{L}$ transgenic and wild-type littermates were immunized subcutaneously in the flanks with $200 \mu \mathrm{g}$ of MOG peptide in $0.1 \mathrm{~mL}$ PBS and $0.1 \mathrm{~mL}$ CFA containing $0.4 \mathrm{mg}$ Mycobacterium tuberculosis (H37Ra; Difco Laboratories, Detroit, Michigan, USA) and intraperitoneally injected with 200 ng Pertussis toxin (List Biological Laboratories Inc., Campbell, California, USA) on the day of immunization and 2 days later.

EAE was scored as previously described $(18,19)$ : grade 1 , limp tail or isolated weakness of gait without limp tail; grade 2, partial hind limb paralysis; grade 3, total hind limb or partial hind and front limb paralysis; grade 4, total hind limb and partial front limb paralysis; grade 5, moribund or dead animal. In C57/B16 mice, MOG-induced disease develops around day 15 (Table 1), and the mice undergo an acute phase lasting 10-12 days. Around 50\% of the mice develop a persistent chronic disease at the end of the acute phase, which is characterized by a disease grade of 1 or more (20).

MBP and PLP immunization. Bcl- $\mathrm{x}_{\mathrm{L}}$ transgenic mice and wild-type littermates were immunized subcutaneously in the flanks with $100 \mu \mathrm{g}$ of mouse myelin basic protein (MBP), prepared as previously described (21), emulsified in an equal amount of CFA containing $2 \mathrm{mg} / \mathrm{mL} M$. tuberculosis (Difco Laboratories). The animals received a 200ng dose of pertussis toxin intraperitoneally (List Biological Laboratories Inc.) 24 hours after immunization. Mice were immunized with proteolipid protein (PLP) (provided by V. Kuchroo, Brigham and Women's Hospital, Boston, Massachusetts, USA) $(100 \mu \mathrm{g}$ per mouse emulsified in an equal amount of CFA containing $4 \mathrm{mg} / \mathrm{mL} M$. tuberculosis). The mice received $100 \mathrm{ng}$ of pertussis toxin intravenously on days 0 and 2 after immunization. Scoring of clinical disease was performed daily as above.

Staining for T-cell receptor variable $\beta$ (TCR V $\beta$ ) chains. Splenocytes from naïve or MOG-immunized mice (on day 12 after immunization) were separated and resuspended

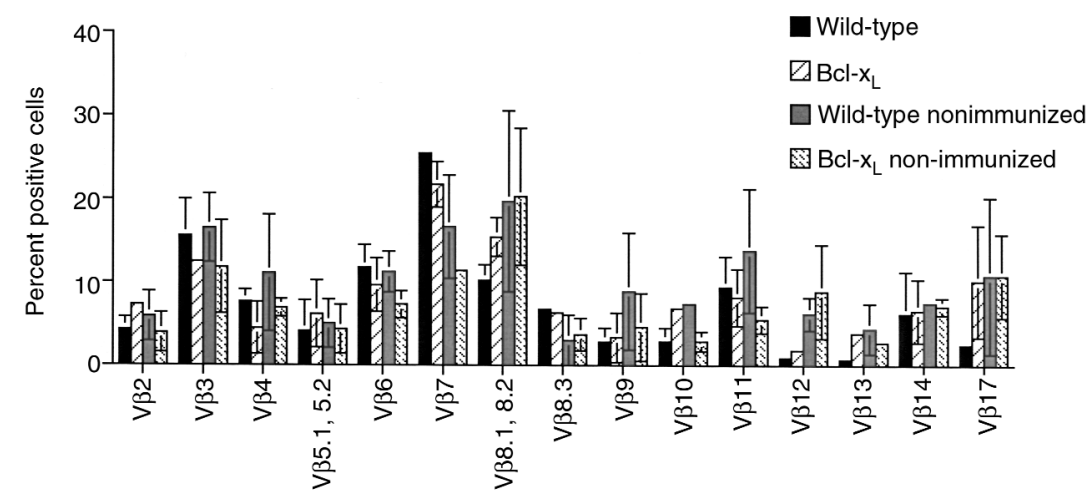

Figure 1

Splenocytes were obtained from naïve or MOG-immunized (day 12) Bcl- $x_{\mathrm{L}}$ and wild-type mice. There were 3 mice per group. Splenocytes were stained with TCR $\beta$ chain constant region and a panel of $V \beta$-specific antibodies. The $y$ axis represents the percentage of T cells expressing a particular $V \beta$ chain $( \pm S D)$. There were no significant differences between the $B c l-x_{L}$ and wild-type splenocytes. 
Figure 2

Draining lymph node cells were obtained on day 10 after immunization from $\mathrm{Bcl}-\mathrm{x}_{\mathrm{L}}$ transgenic (striped bars) and wild-type littermates (black bars). The cells were cultured in the presence of MOG p35-55 at 1,10 , and $100 \mu \mathrm{g} / \mathrm{mL}$ or Con A $(2.5 \mu \mathrm{g} / \mathrm{mL})$ (on the $x$ axis). (a) Stimulation indices after stimulation with MOG $\left({ }^{\mathrm{A}} P=0.04\right.$, ${ }^{\mathrm{B}} P=0.002$ for wild-type versus transgenic). (b) ELISPOT for MOG-specific IFN- $\gamma$-producing cells. The $y$ axis represents the number of positive cells per $10^{5}$ cells plated ( $A P=0.03$ ). (c) IFN$\gamma$ and (d) IL-10 production were measured in the supernatants after 48 hours of culture by ELISA. Cytokine production was significantly higher in the cultures from transgenic mice at all antigen doses $(P<0.0005$ by $t$-test $)$. $\mathbf{a}$

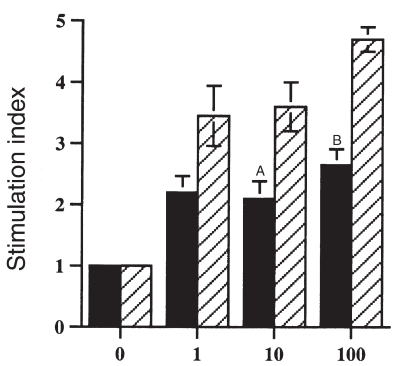

c

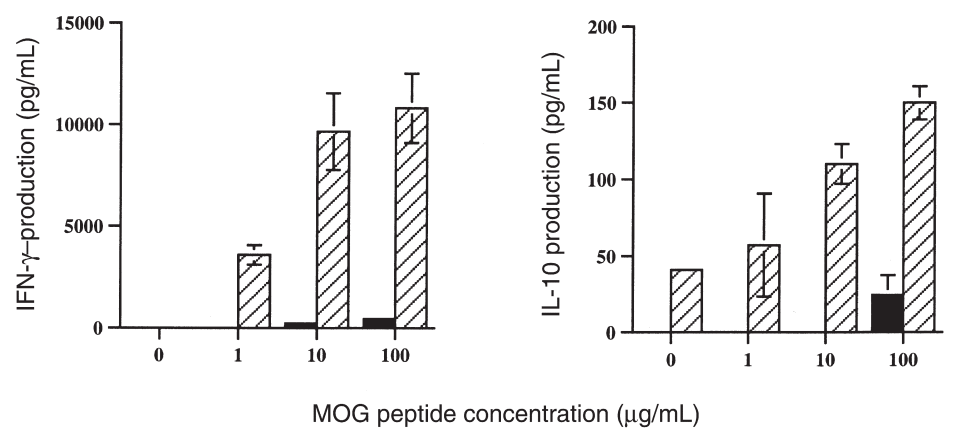

b

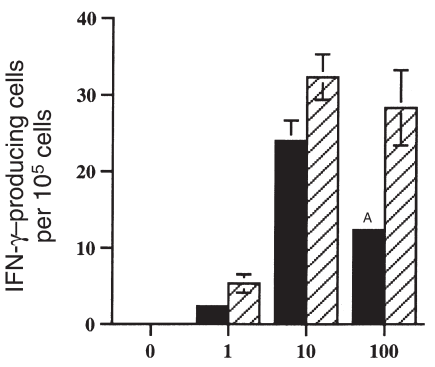

d in PBS to a concentration of $10^{7}$ cells $/ \mathrm{mL}$. Next, $100-\mu \mathrm{L}$ aliquots were plated in a 96-well plate. The cells were treated with Fc Block (PharMingen, San Diego, California, USA) at a concentration of $1 \mu \mathrm{g} / 10^{6}$ cells for 5 minutes on ice. The cells were then stained with various V $\beta$ FITC- or PE-conjugated antibodies (PharMingen): V $\beta 2$ rat antimouse IgG2a PE, V 33 hamster anti-mouse IgG PE, V $\beta 4$ rat anti-mouse IgG2b PE, V $\beta 5.1,5.2$ mouse anti-mouse IgG1 PE, V $\beta 6$ rat anti-mouse IgG2b PE, V $\beta 7$ rat antimouse IgG2b PE, V $\beta 8.1$, 8.2 mouse anti-mouse IgG2a FITC, V $\beta 8.3$ hamster anti-mouse IgG PE, V $\beta 9$ mouse antimouse IgG1 FITC, V $\beta 10$ rat anti-mouse IgG2a PE, V $\beta 11$ rat anti-mouse IgG2b PE, V $\beta 12$ mouse anti-mouse IgG1 FITC, V $\beta 13$ mouse anti-mouse IgG1 PE, V $\beta 14$ rat antimouse IgM FITC, V $\beta 17$ mouse anti-mouse IgG2a, and TCR $\beta$ chain constant region hamster anti-mouse FITC and PE. Appropriate isotype controls were used.

The cells were incubated on ice for 30 minutes, washed with $1 \%$ FBS in PBS twice, and then resuspended for analysis on a FACScan equipped with CellQuest software (both from Becton Dickinson Immunocytometry Systems, San Jose, California, USA). Cells expressing specified V $\beta$ type were plotted against the TCR constant region on dot plot, and percentages were calculated.

Cell culture. For in vitro cell culture experiments, mice were subcutaneously immunized in 1 hind footpad and in both flanks with an emulsion of $100 \mu \mathrm{L}$ CFA and $100 \mu \mathrm{L}$ PBS containing $100 \mu \mathrm{g}$ MOG p35-55. The mice were sacrificed 10 days after immunization.

A single cell suspension was prepared from the inguinal and the draining popliteal lymph nodes or spleens. For proliferation and cytokine measurement, the cells were cultured in 96-well plates (Costar, Corning, New York, USA). Media used for proliferation and cytokine assays consisted of serum-free Ex-Vivo 20 medium (BioWhittaker Inc., Walkersville, Maryland, USA) containing $75 \mathrm{mM} / \mathrm{mL}$-glutamine, $100 \mathrm{U} / \mathrm{mL}$ penicillin and streptomycin, $1 \mathrm{~mL} / 100 \mathrm{~mL}$ of a $\times 100$ concentrated non-essential amino acid solution, 0.1 $\mathrm{mM}$ HEPES $/ \mathrm{mL}, 1 \mathrm{mM} / \mathrm{mL}$ sodium pyruvate (all from BioWhittaker Inc.), and $0.05 \mathrm{mM} / \mathrm{mL}$ 2-mercaptoethanol (Sigma Chemical Co.). Cells were incubated at $37^{\circ} \mathrm{C}$ in humidified air containing $7 \% \mathrm{CO}_{2}$.

Proliferation assay. The cells were cultured at $2 \times 10^{6}$

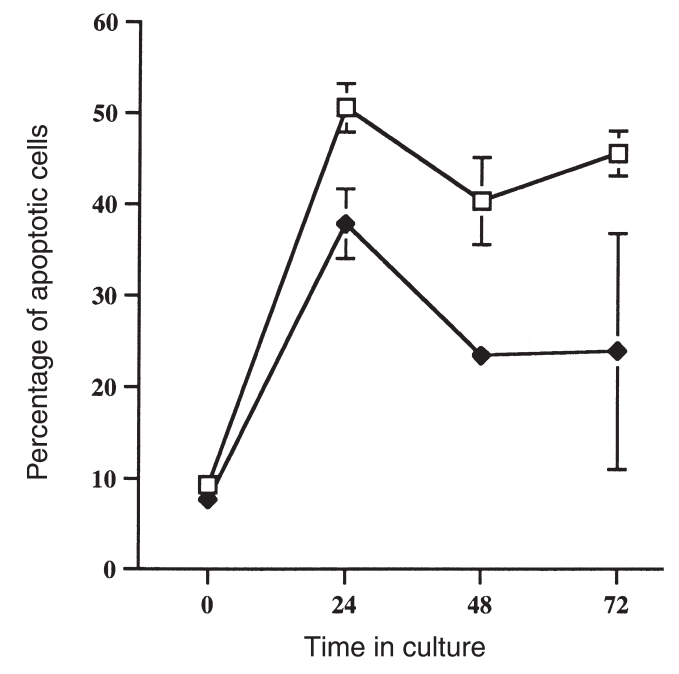

\section{Figure 3}

Quantitation of apoptotic cells during in vitro culture. Primed lymph node cells were collected from the draining lymph nodes of mice immunized with MOG p35-55 10 days earlier. The cells were cultured with MOG p35-55 at $10 \mu \mathrm{g} / \mathrm{mL}$ and collected for staining at the times indicated on the $x$ axis. The percentage of apoptotic cells was determined by TUNEL staining of CD4 cells. The data are the mean of 2 experiments. There were significant differences between the 2 groups ( $P=0.0081$ by repeated measures ANOVA). 
cells/mL and $200 \mu \mathrm{L} /$ well with various antigen concentrations. After 48 hours of culture, $1 \mu \mathrm{Ci}\left[{ }^{3} \mathrm{H}\right]$ thymidine (NEN Life Science Products Inc., Boston, Massachusetts, USA) was added in $10 \mu \mathrm{L}$ of media to each well for another 16 hours. Cells were harvested with a Wallac 1205 Betaplate Scintillation Counter (Wallac Oy, Turku, Finland) on filter mats and were then dried and counted.

Cytokine ELISA. The cells were cultured at $2 \times 10^{6}$ cells $/ \mathrm{mL}$ in $200 \mu \mathrm{L}$ media at various antigen concentrations. Supernatants for IL-10 and IFN- $\gamma$ ELISA were collected after 48 hours of culture. Quantitative ELISAs for IL-10 and IFN- $\gamma$ were performed using paired antibodies and recombinant cytokines from PharMingen according to the manufacturer's recommendations.

Staining for apoptosis of lymphocytes. Cells were cultured in DMEM complete at a concentration of $2 \times 10^{6}$ cells/mL with MOG p35-55 at a concentration of 10 $\mu \mathrm{g} / \mathrm{mL}$ in 6 -well plates. The cells were harvested for TdT-mediated dUTP nick-end labeling (TUNEL) staining at $0,24,48$, and 72 hours. The cells were washed and resuspended in PBS to a concentration of $10^{7} / \mathrm{mL}$, and then incubated on ice with rat anti-mouse CD4 PE-conjugated antibody (Caltag Laboratories Inc., Burlingame, California, USA) for 20 minutes. They were then washed with PBS and fixed in 4\% paraformaldehyde for $30 \mathrm{~min}$ utes at room temperature. The cells were washed and permeabilized with $100 \mu \mathrm{L}$ Triton X-100 in $0.1 \%$ sodi-

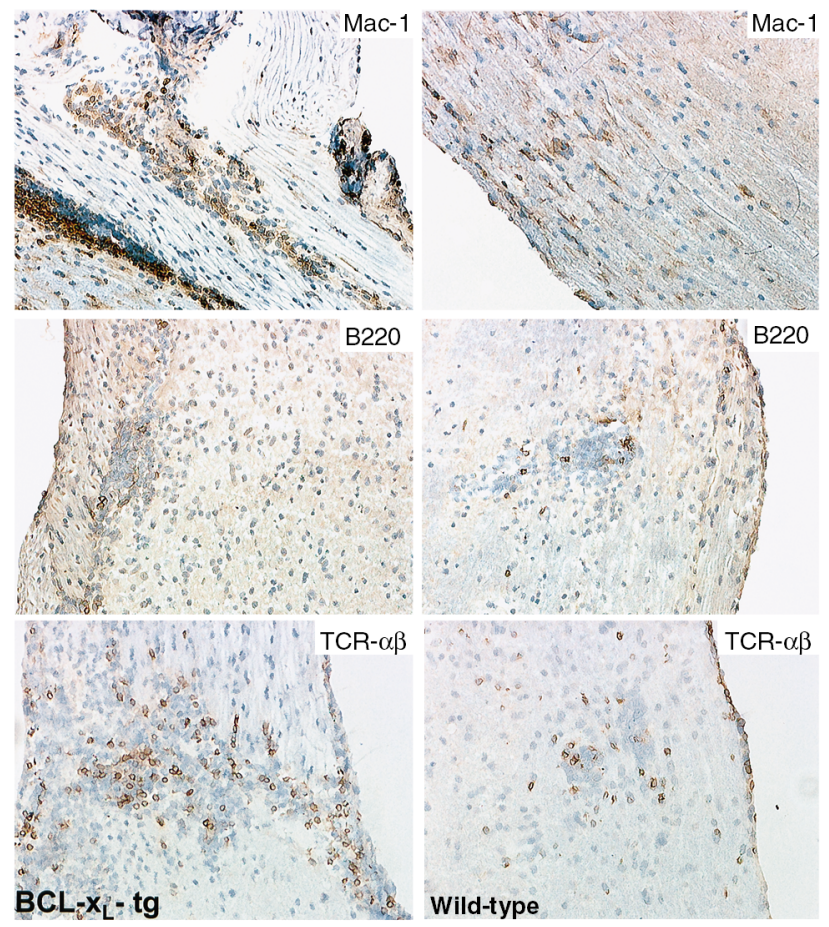

\section{Figure 4}

Microphotograph (magnification $\times 200$ ) of representative spinal cord sections from wild-type and $\mathrm{Bcl}-\mathrm{x}_{\mathrm{L}}$ transgenic mice ( $n=4$ per group) after immunization with $200 \mu \mathrm{g}$ of MOG p35-55. The spinal cords were collected when mice reached grade 2 . The sections were immunohistochemically stained for activated macrophages (Mac-1), $\mathrm{B} 220^{+} \mathrm{B}$ cells (B220), and $\alpha \beta \mathrm{TCR}^{+}$cells. Cells positive for the marker indicated display a brown color.

\section{Table 1}

Characteristics of the disease in $\mathrm{Bcl}-\mathrm{x}_{\mathrm{L}}$ transgenic and wild-type littermates $^{A}$

\begin{tabular}{lcc}
\hline & Control & $B C l-x_{\mathrm{L}}$ \\
Incidence & $23 / 26$ & $26 / 26$ \\
Mean maximal grade \pm SEM & $1.5 \pm 0.3$ & $2.2 \pm 0.3^{\mathrm{B}}$ \\
Day of onset \pm SEM & $15.7 \pm 0.9$ & $12.6 \pm 0.6^{\mathrm{C}}$ \\
Proportion with chronic EAE & $11 / 26$ & $18 / 26^{\mathrm{E}}$ \\
Deaths & $1 / 26$ & $5 / 26$
\end{tabular}

AC Composite data from 4 experiments $\left(n=26\right.$ per group). ${ }^{\mathrm{B}} P=0.03$ by MannWhitney $U$ test. ${ }^{C} P=0.006$ by Mann-Whitney $U$ test. ${ }^{D}$ Chronic phase was considered to start on day 25; this is the number of animals with a higher grade than 1 after the acute phase. $E P=0.046$ by Fisher's exact test.

um citrate for 2 minutes on ice. The cells were then washed, and the positive controls were treated with DNase (GIBCO BRL, Gaithersburg, Maryland, USA) for 10 minutes at room temperature. After another wash, the cells were incubated with TUNEL label plus enzyme (Roche Molecular Biochemicals, Indianapolis, Indiana, USA) for 1 hour at $37^{\circ} \mathrm{C}$. Cells were then washed and analyzed by flow cytometry on a FACSort equipped with CellQuest software (both from Becton Dickinson Immunocytometry Systems).

We also used 7-amino-actinomycin D (7-AAD) staining (Calbiochem-Novabiochem Corp., La Jolla, California, USA) to measure apoptosis in vitro. Staining of apoptotic cells with 7-AAD was done as described by Schmid et al. (22). Briefly, cells were prestained for surface antigen expression as above, were incubated with $20 \mu \mathrm{g} / \mathrm{mL}$ of $7-A A D$ in PBS-Az for 20 minutes at $4^{\circ} \mathrm{C}$, and then were protected from light. Cells were then analyzed on FACSort (Becton Dickinson Immunocytometry Systems) without further washing. Staining with 7-AAD differentiates cells with 7-AAD dim that are in the early stages of apoptosis and 7-AAD ${ }^{\text {bright }}$ that are in the later stages of apoptosis (22).

ELISPOT assay. Cells $\left(4 \times 10^{6} /\right.$ well $)$ were incubated with antigen (as for the proliferation assays) in U-bottom plates for 24 hours. The cells were then counted, resuspended, and serially diluted from a concentration of $10^{6}$ cells $/ \mathrm{mL}$ down to $8 \times 10^{3}$ cells $/ \mathrm{mL}$, and then were added to nitrocellulose plates (Millipore, Bedford, Massachusetts, USA). The plates were coated with $50 \mu \mathrm{L}$ primary IFN- $\gamma \mathrm{Ab}$ (clone R4-6A2; Endogen Inc., Woburn, Massachusetts, USA) at a concentration of $5 \mu \mathrm{g} / \mathrm{mL}$. Next, 100 $\mu \mathrm{L}$ of the appropriate concentrations of antigen or mitogen was added to the nitrocellulose ELISPOT plates. The cells were then incubated for an additional 18 hours at $37^{\circ} \mathrm{C}$. After washing three times, $50 \mu \mathrm{L} /$ well of biotinylated IFN- $\gamma$ secondary Ab (clone XMG1.2; Endogen Inc.) was added to the plates at a concentration of $2 \mu \mathrm{g} / \mathrm{mL}$ diluted in 1\% BSA/PBS for 5 hours at room temperature. After washing, $50 \mu \mathrm{L}$ of alkaline phosphatase (E-2636; Sigma Chemical Co.) was added to the plates at a dilution of 1:1,000 in $0.05 \%$ Tween/PBS for 2 hours at room temperature. Plates were washed twice with wash buffer and then twice in PBS. A 50- $\mu \mathrm{L}$ solution of BCIP/NBT (FASTR B-5655; Sigma Chemical Co.) was added for 
5-20 minutes until blue spots developed. Plates were washed with distilled water. Spots were counted using a Stemi 100 dissecting microscope (Carl Zeiss Inc., Thornwood, New York, USA).

Antibodies. The following antibodies were obtained from PharMingen: purified rat IgG1, IgG2a, and IgG2b, purified hamster IgG, rat anti-mouse CD11b, rat anti-mouse CD45R/B220, rat anti-mouse IFN- $\gamma /$ R4-6A2, rat antimouse IL4/BVD4-1D11, rat anti-mouse IL-10/JES5-2A5, rat anti-mouse IL-12/C17.8, rat anti-mouse TNF- $\alpha /$ MP6XT22, and rat anti-mouse macrophage clone F4/80 (Caltag Laboratories Inc.). Biotinylated swine anti-rabbit Ig was obtained from DAKO A/S (Glostrup, Denmark), and biotinylated anti-hamster IgG and biotinylated anti-rat IgG were purchased from Vector Laboratories (Burlingame, California, USA).

Immunohistology. Spinal cords and brains were collected at the peak of disease (as mice reach grade 2) from 4 to 8 mice in each experimental group. Spinal cord tissues were embedded in OCT Compound (Tissue-Tek, Sakura Finetek, Torrance, California, USA), quick frozen in liquid nitrogen, and kept at $-70^{\circ} \mathrm{C}$ until sectioning. Cryostat sections $(10 \mu \mathrm{m})$ of spinal cords were fixed with acetone or $4 \%$ paraformaldehyde and then labeled with the antibody of interest. The sections were stained using the avidin-biotin technique (Vectastain Elite kit; Vector Laboratories), visualized with diamino-benzidine (Vector Laboratories), and then counterstained in hematoxylin. Isotype-matched Ig and omission of the primary antibody served as negative controls. Each specimen was evaluated at least at 3 different levels of sectioning. The whole tissue section (a longitudinal spinal cord section) was evaluated for a given cellular marker at $\times 40$ magnification, and the results were expressed as mean number of labeled cells per $100 \mathrm{~mm}^{2}$ of spinal cord tissue. Evaluation of cytokine expression was done semiquantitatively because of the tendency of cytokines to diffuse around cells. The following arbitrary scale was used: -, no positive cells; +, 1-9 positive cells; ++, 10-99 positive cells; +++, 100-1,000 positive cells; and ++++, if positive cells exceeded 1,000 per $100 \mathrm{~mm}^{2}$ of tissue examined.

To assess demyelination, spinal cords were collected on days 10 and 13 after immunization, and quick-frozen sections were stained with the Luxol fast blue method. The sizes of demyelinating areas were measured throughout the sections by using KS 400 image analysis system (Carl Zeiss AB, Stockholm, Sweden), the whole tissue was then measured, and the demyelinating areas were calculated and expressed per $100 \mathrm{~mm}^{2}$ of spinal cord tissue.

TUNEL staining to measure apoptosis in the CNS. TUNEL staining was applied to assess the number of apoptotic cells in CNS tissues. CNS tissue samples were obtained on days 13-15, 20, and 50 after immunization. Briefly, CNS tissues were quick frozen as above, $10-\mu \mathrm{m}$ cryosections were then fixed with $4 \%$ paraformaldehyde and treated with $0.1 \%$ of Triton X-100 (Sigma Chemical Co.). TUNEL labeling was performed according to the manufacturer's recommendations using a commercial kit pur-

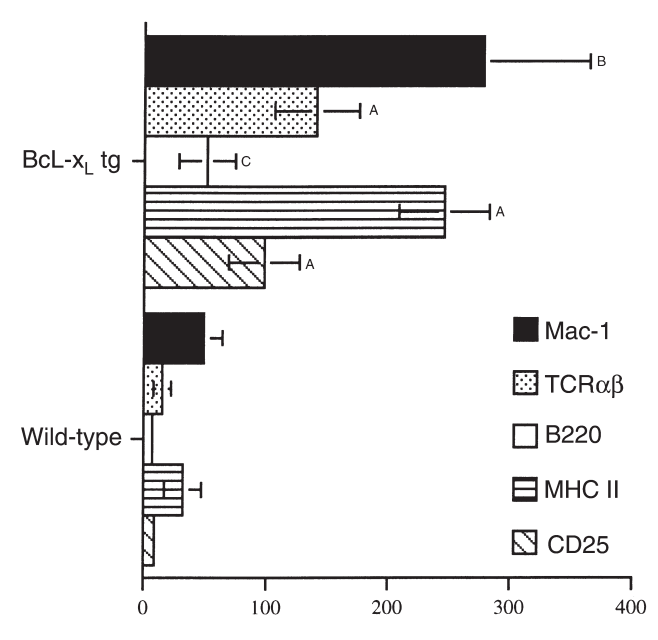

Number of positive cells $/ 100 \mathrm{~mm}^{2}$ of spinal cord

\section{Figure 5}

Quantitation of cellular infiltrates in the spinal cords of wild-type and $\mathrm{Bcl}-\mathrm{x}_{\mathrm{L}}$ transgenic mice. The cords were collected on day 13 after immunization and stained with markers for macrophages (Mac-1), T cells (TCR $\alpha \beta$ ), B cells (B220), class II MHC (MHC II), and IL-2R (CD25). The mean number of labeled cells per $100 \mathrm{~mm}^{2}$ of spinal cord tissue was calculated for each marker using the KS 400 image analysis system (Carl Zeiss). The $x$ axis represents the number of cells per $100 \mathrm{~mm}^{2}$ of tissue. There were significant differences between the 2 groups in all the markers listed ( ${ }^{A} P<0.0001,{ }^{B} P=0.0004,{ }^{C} P=$ 0.0028 for B220 by $t$ test).

chased from Boehringer Mannheim GmbH (Mannheim, Germany). Fluorescein-labeled cells were counted at $\times 20$ magnification using a fluorescence microscope (Carl Zeiss) throughout the sections. The total area of the sections was measured, and the number of positive cells were expressed per $100 \mathrm{~mm}^{2}$ of tissue area using a KS 400 image analysis system (Zeiss Swedish AB).

\section{Results}

$B c l-x_{L}$ transgenic mice have a more severe disease induced by MOG peptide. Bcl- $\mathrm{x}_{\mathrm{L}}$ transgenic mice have a more severe form of EAE after active immunization with MOG p35-55 than wild-type animals. Table 1 shows the composite data of 4 experiments. There was a significant difference in the mean maximal grade $(P=0.03)$ and the day of onset $(P=0.006)$ between the 2 groups, both of

\section{Table 2}

Extent of demyelination in the CNS on day 10 and day 13 after immunization

$\begin{array}{lcc}\text { Groups } & \text { Area of demyelination }\left(\mathrm{mm}^{2}\right) / 100 \mathrm{~mm}^{2} \text { of spinal cord } \\ & \text { Day } 10 \text { after immunization } & \text { Day } 13 \text { after immunization } \\ \text { Wild-type } & 0.1 \pm 0.1 & 7.8 \pm 0.9 \\ \text { Bcl- } x_{\mathrm{L}} & 15.2 \pm 1.4^{\mathrm{A}} & 60 \pm 3.2^{\mathrm{A}}\end{array}$

Mice were immunized with MOG p35-55. One group of mice was sacrificed on day 10 and another on day 13 after immunization; the spinal cords were dissected and examined. Demyelination was assessed quantitatively at 3 different levels of sectioning. The numbers depicted here show mean values of demyelinated areas $\left(\mathrm{mm}^{2}\right)$ per $100 \mathrm{~mm}^{2}$ of tissue per each group (3 mice per group) $( \pm \mathrm{SE}) .{ }^{A} P<0.0001$ by Mann-Whitney $U$ test. 


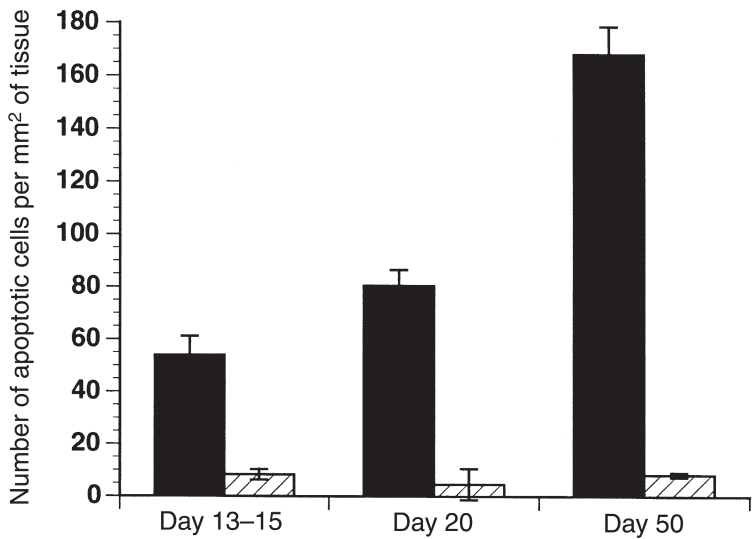

Figure 6

Quantitation of TUNEL-positive cells in the CNS of Bcl- $x_{\mathrm{L}}$ and wildtype mice. CNS tissue samples were obtained on days 13-15, 20, and 50 after immunization. Fluorescein-labeled cells were counted at $\times 20$ magnification throughout the sections, the total area of the sections was measured, and the number of positive cells was expressed per $100 \mathrm{~mm}^{2}$ of tissue area. There were significant differences in the number of apoptotic cells at all time points (days 13-15, $P=0.004$; day $20, P=0.0002$; day $50, P=0.0001$ )

these measures reflect differences in the acute disease. Interestingly, there was also a difference in the chronic phase of the disease: in Bcl- $\mathrm{x}_{\mathrm{L}}$ transgenic animals, the proportion of mice with chronic disease (mice with grade 1 or more) was significantly higher $(P=0.04)$.

Susceptibility of Bcl- $x_{L}$ transgenic mice to develop severe EAE is not due to a broader autoreactive T-cell repertoire. C57BL/6 mice are highly resistant to EAE induction by other myelin antigens such as MBP and PLP. To address the question of whether the expression of the $\mathrm{Bcl}-\mathrm{x}_{\mathrm{L}}$ transgene renders the mice susceptible to disease induced by other autoantigens, we immunized both Bcl- $\mathrm{x}_{\mathrm{L}}$ transgenic and wild-type mice with MBP or PLP and checked for EAE development. Both Bcl- $\mathrm{x}_{\mathrm{L}}$ transgenic mice and wild-type mice developed very mild EAE after MBP or PLP immunization, which was manifested by slight tail weakness. These results indicate that transgenic expression of the long form of the $b c l$ $x$ gene targeted to the T-cell lineage does not alter the Tcell repertoire or broaden the pool of T cells capable of mediating EAE. After staining naïve splenic $\mathrm{T}$ cells or splenocytes from MOG-immunized mice with a panel of antibodies against $V \beta$ chain of TCR, we found no significant differences in $\mathrm{V} \beta$ usage between $\mathrm{Bcl}-\mathrm{x}_{\mathrm{L}}$ transgenic and wild-type littermates (Figure 1).

$B c l-x_{L}$ transgenic mice have increased T-cell responses and improved survival after MOG $p 35-55$ stimulation in vitro. As shown in Figure 2a, there was a significant increase in the in vitro proliferation $(P=0.04$ for MOG $10 \mu \mathrm{g} / \mathrm{mL}$ and $P=0.002$ for MOG $100 \mu \mathrm{g} / \mathrm{mL}$ ) in draining lymph node cultures from Bcl- $\mathrm{x}_{\mathrm{L}}$ transgenic animals as compared with wild-type mice immunized with MOG p35-55. The precursor frequency of cells reacting to MOG was estimated by ELISPOT with the number of IFN- $\boldsymbol{\gamma}$-secreting cells after MOG stimulation. There were more MOG-reactive IFN- $\boldsymbol{\gamma}$-secreting cells in the cultures from $\mathrm{Bcl}-\mathrm{x}_{\mathrm{L}}$ mice, reaching significance at the highest antigen dose $(P=0.03)$. Figure $2 \mathrm{~b}$ shows the number of MOG-reactive cells per $10^{5}$ cells plated. The number of cells decreased proportionately as the number of cells plated decreased, thus confirming the specificity of the response. There was no difference in Con A-stimulated IFN- $\gamma$-producing cells between wild-type and transgenic animals (not shown). Cytokine secretion was also significantly increased during in vitro stimulation with antigen (Figure 2, c and d), and cytokine secretion after Con A stimulation was similar between the 2 groups (not shown).

To determine if cells from $\mathrm{Bcl}-\mathrm{x}_{\mathrm{L}}$ transgenic mice had improved survival in vitro, we used a TUNEL-staining method for flow cytometry. Primed lymph node cells were cultured in vitro with MOG p35-55, and the percentage of apoptotic cells in the culture was determined at various time points. As seen in Figure 3, the percentage of apoptotic cells (TUNEL-positive cells) was persistently higher in the control cultures when compared with the cultures from $\mathrm{Bcl}-\mathrm{x}_{\mathrm{L}}$ transgenic mice $(P=0.0081$ by repeated measures ANOVA); this was similar to our findings with antigen nonspecific stimulation (17). These findings were confirmed by measuring the percentage of $7 \mathrm{AAD}^{\text {lo }}$ cells in the cultures, because $7 \mathrm{AAD}^{\text {lo }}$ population stains apoptotic cells, whereas 7AAD high stains dead cells (22).

$B c l-x_{L}$ transgenic mice have more extensive CNS inflammation and demyelination. There were marked differences in the histopathologic manifestation of EAE in the CNS of $\mathrm{Bcl}-\mathrm{x}_{\mathrm{L}}$ transgenic mice when compared with wild-type mice, which were more prominent than the differences in clinical disease. Bcl- $x_{\mathrm{L}}$ transgenic mice exhibited extensive demyelination on day 10 after immunization in both the spinal cord and brain, whereas wild-type mice showed no evidence of demyelination on day 10. On day 13 after immunization, both groups of mice had areas of demyelination, but it was more extensive in the CNS of Bcl- $x_{\mathrm{L}}$ compared with wild-type mice (Table 2). The sections from Bcl- $x_{\mathrm{L}}$ mice showed $64 \pm 9 \mathrm{~mm}^{2}$ of demyelination per $100 \mathrm{~mm}^{2}$ of tissue compared with 15 $\pm 4 \mathrm{~mm}^{2}$ in the wild-type CNS. Furthermore, $\mathrm{Bcl}-\mathrm{x}_{\mathrm{L}}$ transgenic sections showed massive inflammation and inflammatory infiltrates consisting of activated macrophages/microglia $\left(\mathrm{Mac}-1^{+}\right)$, B cells, and activated T cells $\left(\mathrm{CD} 25^{+}\right)$(Figures 4 and 5). Furthermore, investigation of cytokine production in the CNS showed that $\mathrm{Bcl}-\mathrm{x}_{\mathrm{L}}$ transgenic mice had more IFN- $\gamma$, IL-12, and TNF$\alpha$ production in the CNS when compared with wildtype mice (Table 3 ). There were no significant differences in IL-4 and IL-10 in the CNS.

$B c l-x_{L}$ transgenic mice have less apoptotic cells in the infiltrates. To investigate whether protection from apoptosis contributed to the worsening of EAE, we compared the number of apoptotic cells in the CNS of transgenic mice and littermates. TUNEL staining of spinal cord tissues was performed on samples taken on days 13-15, 20, and 50. At all time points tested there was a 
decreased number of apoptotic cells in the CNS infiltrates of Bcl- $x_{\mathrm{L}}$ transgenic mice compared with wildtype mice. $\mathrm{Bcl}-\mathrm{x}_{\mathrm{L}}$ transgenic mice had only occasional cells staining by TUNEL compared with the wild-type mice. Quantitation of the TUNEL-positive cells in the CNS showed significantly less apoptotic cells in the transgenic mice at all time points (Figure 6). Interestingly, the number of apoptotic cells in the wild-type control animals increased significantly between days 13 and $50(P=0.0001)$, whereas there was no change in the number of apoptotic cells in the Bcl- $x_{L}$ mice, suggesting a link between apoptosis and recovery from disease.

\section{Discussion}

$\mathrm{Bcl}-2$ and related proteins such as $\mathrm{Bcl}-\mathrm{x}_{\mathrm{L}}$ are products of a family of genes that modulate programmed cell death or apoptosis. The function of these genes is critical not only during development and tissue homeostasis, but also in the pathogenesis of a variety of diseases including autoimmune diseases, neurodegenerative disorders, cancer, and viral infections. A failure to undergo apoptosis or programmed cell death may contribute to the pathogenesis of these diseases. In EAE, clinical remissions and recovery from clinical symptoms may be related to inflammatory cell death through apoptosis. The role of activation-induced cell death though the Fas/FasL pathway has been investigated in $\operatorname{EAE}(11,12)$, but the role of passive cell death is unknown. Here we address the role of T-cell apoptosis by passive cell death in EAE. We used mice with transgenic expression of the long form of the $b c l-x$ gene targeted to the T-cell lineage $(3,4)$. In these mice, $\mathrm{Bcl}-\mathrm{x}_{\mathrm{L}}$ is constitutively expressed in $T$ cells and enhances their survival when cultured in vitro. We found that mice transgenic for $\mathrm{Bcl}-\mathrm{x}_{\mathrm{L}}$ have an earlier onset and a more severe form of EAE induced by MOG p35-55 when compared with wild-type C57BL/6 mice and nontransgenic littermates.

The differences in pathology between transgenic and wild-type animals were even more prominent than the differences in clinical disease scores. This may reflect increased survival of the activated cells in the CNS, thus leading to a persistent chronic disease in the transgenic mice. The persistent $\mathrm{T}$ cells could also be providing more help for $\mathrm{B}$ cells even though the transgene is expressed in $\mathrm{T}$ cells. This is consistent with the increased demyelination seen in the Bcl- $x_{\mathrm{L}}$ mice. Alternatively, the increased severity of EAE in Bcl- $\mathrm{x}_{\mathrm{L}}$ transgenic mice could be the result of broader autoreactive T-cell repertoire. Although it was shown that the $b c l-x_{L}$ gene does not prevent clonal deletion of autoreactive thymocytes (3), we questioned whether peripheral mechanisms of tolerance to self-reactive thymocytes that escaped central selection were disrupted by the transgenic expression of $\mathrm{Bcl}-\mathrm{x}_{\mathrm{L}}$. Thus, we immunized $\mathrm{Bcl}-\mathrm{x}_{\mathrm{L}}$ transgenic and wild-type mice with other myelin antigens, MBP and PLP, and found that immunization with either of these did not induce significant clinical EAE in Bcl- $\mathrm{x}_{\mathrm{L}}$ transgenic or wild-type mice. Thus, the increased severity of EAE in Bcl- $x_{\mathrm{L}}$ transgenic mice is

\section{Table 3}

Cytokine expression in the CNS at the peak of disease

$\begin{array}{lcccc} & \text { IFN- } \gamma & \text { TNF- } \alpha & \text { IL-10 } & \text { IL-4 } \\ \text { Wild-type } & + & + & + & + \\ \text { Bcl-x } \mathrm{L}^{+/+} & ++ & +++ & + & +\end{array}$

Semiquantitative assessment of immunohistochemical staining for IFN- $\gamma$, TNF$\alpha, \mathrm{IL}-10$, and IL-4 around the cellular infiltrates in spinal cord tissues from wildtype and Bcl- $x_{L}$ transgenic animals after immunization with MOG p35-55. The tissues were collected at the peak of clinical EAE on day 13 after immunization. Positive staining was measured semiquantitatively as follows:,$+ 1-10$ positive cells;,$++ 11-100$ positive cells; +++, more than 100 positive cells.

likely due to increased survival of MOG-reactive $\mathrm{T}$ cells and/or to an increased capacity to proliferate and produce inflammatory cytokines. Hence, we studied the capacity of Bcl- $x_{\mathrm{L}}$ transgenic $\mathrm{T}$ cells to proliferate and investigated their cytokine production.

Previous studies from our group with nonantigen specific stimulation, showed that $\mathrm{Bcl}-\mathrm{x}_{\mathrm{L}}$ transgenic cells had fewer cell divisions than non-transgenic cells (17). However, our in vitro studies showed increased proliferation and cytokine production of peripheral antigen-specific $T$ cells from Bcl- $x_{\mathrm{L}}$ transgenic mice when compared with wild-type mice in response to MOG p35-55. This would suggest that the precursor frequency of MOG-specific $\mathrm{T}$ cells is higher in the lymph node cells from Bcl- $x_{L}$ transgenic mice, probably the result of enhanced survival of these cells after priming. Consistent with this data, we found an increased number of MOG-specific IFN$\gamma$-secreting cells and enhanced survival of primed lymph node cells in vitro after stimulation with MOG peptide.

The clinical manifestations of disease correlated with increased inflammatory infiltrates in the CNS and increased local production of IFN- $\gamma$ and TNF- $\alpha$. Furthermore, there were more areas of demyelination in the $\mathrm{Bcl}-\mathrm{x}_{\mathrm{L}}$ transgenic mice suggesting that the increased infiltrates translate to worsening pathology. TUNEL staining of the CNS during the peak of EAE showed less apoptotic cells in the transgenic mice compared with littermates. Furthermore, in the wild-type animals the number of apoptotic cells increased during the course of the disease, which is consistent with previous reports (23-25). Previous studies in the Lewis rat model reveal that during spontaneous recovery from EAE autoreactive $\mathrm{T}$ cells are eliminated from the CNS by activationinduced apoptosis involving the Fas pathway $(23,24)$. There are several pathways leading to elimination of $\mathrm{T}$ cells by apoptosis. Fas-mediated apoptosis may be crucial in the Lewis rat model, whereas passive cell death may be more important in the murine model, as shown by our data. In transplantation tolerance, both passive cell death and AICD appear crucial for tolerance induction (17). These results underscore the crucial role of Tcell apoptosis in the outcome of an autoimmune attack and indicate that termination of a $\mathrm{T}$ cell-mediated autoimmune reaction is dependent on clearance of autoreactive cells through programmed cell death. Infiltrating $\mathrm{CD}^{+} \mathrm{T}$ cells and parenchymal microglia express 
Fas, FasL, and Bax in CNS, and the upregulation of these molecules is associated with disease activity (26). The role of Fas/FasL pathway in regulation of autoimmune responses remains controversial. Apoptosis mediated by Fas/FasL interaction was thought to be critical in development of EAE because mice with mutations in Fas or FasL expression are resistant to $\operatorname{EAE}(11,12)$.

More recently, studies in Fas-deficient SJL (SJL lpr/lpr) mice showed that even though the incidence of the disease is lower than that in heterozygous littermates, the disease was more severe and less likely to remit (13). The studies in these Fas/FasL-deficient models are complicated by the fact that the mutation is not restricted to the T-cell lineage, and apoptosis of microglia cells and possibly oligodendrocytes is also impaired. Fas may play an important role in mediating autoimmune tissue destruction. Upregulation of FasL on activated autoreactive $T$ cells, together with upregulation of Fas on resident cells in the target organ may lead to direct target destruction. Thus, the absence of programmed cell death of microglial and oligodendrocyte may contribute to the resistance to EAE in these models.

By using a system where the antiapoptotic gene $\left(\mathrm{Bcl}-\mathrm{x}_{\mathrm{L}}\right)$ is expressed exclusively on T cells, we could address the role of T-cell apoptosis in termination of neuroinflammation. We conclude that the survival of autoreactive $T$ cells expressing the $b c l-x_{L}$ gene plays a critical role in the pathogenesis of EAE. These findings have implications for understanding the pathogenesis of multiple sclerosis as well as for other autoimmune diseases.

\section{Acknowledgments}

Supported by research grants from the National Multiple Sclerosis Society (RG-2589-A-2 to S.J. Khoury, and FG 1287-A-1 to T. Chitnis), National Institutes of Health (AI40945 to S.J. Khoury, and PO1 AI-41521 to L.A. Turka and M.H. Sayegh), and Fundaçao de Amparo a pesquisa Do Estado De Sao Paulo, Brazil (to K. Abdallah).

1. Van Parijs, L., and Abbas, A.K. 1998. Homeostasis and self-tolerance in the immune system: turning lymphocytes off. Science. 280:243-248.

2. Schmied, M., et al. 1993. Apoptosis of T lymphocytes in experimental autoimmune encephalomyelitis. Evidence for programmed cell death as a mechanism to control inflammation in the brain. Am. J. Pathol. 143:446-452.

3. Grillot, D.A., Merino, R., and Nunez, G. 1995. Bcl-XL displays restricted distribution during $\mathrm{T}$ cell development and inhibits multiple forms of apoptosis but not clonal deletion in transgenic mice. J. Exp. Med. 182:1973-1983.

4. Boise, L.H., et al. 1993. bcl-x, a bcl-2-related gene that functions as a dominant regulator of apoptotic cell death. Cell. 74:597-608.

5. Hockenbery, D., Nunez, G., Milliman, C., Schreiber, R.D., and Korsmeyer, S.J. $1990 . \mathrm{Bcl}-2$ is an inner mitochondrial membrane protein that blocks programmed cell death. Nature. 348:334-336.

6. Nunez, G., Merino, R., Grillot, D., and Gonzalez-Garcia, M. 1994. Bcl-2 and Bcl-x: regulatory switches for lymphoid death and survival. Immunol. Today. 15:582-588.

7. Schwartz, R.H. 1992. Costimulation of T lymphocytes: the role of CD28, CTLA-4, and B7/BB1 in interleukin-2 production and immunotherapy. Cell. 71:1065-1068

8. Van Parijs, L., Peterson, D.A., and Abbas, A.K. 1998. The Fas/Fas ligand pathway and $\mathrm{Bcl}-2$ regulate $\mathrm{T}$ cell responses to model self and foreign antigens. Immunity. 8:265-274.

9. Van Parijs, L., Biuckians, A., and Abbas, A.K. 1998. Functional roles of Fas and Bcl-2-regulated apoptosis of $\mathrm{T}$ lymphocytes. J. Immunol. 160:2065-2071.

10. Lenardo, M. 1991. Interleukin-2 programs mouse $\alpha \beta$ T lymphocytes for apoptosis. Nature. 353:858-861.

11. Sabelko, K.A., Kelly, K.A., Nahm, M.H., Cross, A.H., and Russell, J.H. 1997. Fas and Fas ligand enhance the pathogenesis of experimental allergic encephalomyelitis, but are not essential for immune privilege in the central nervous system. J. Immunol. 159:3096-3099.

12. Waldner, H., Sobel, R.A., Howard, E., and Kuchroo, V.K. 1997. Fas- and FasL-deficient mice are resistant to induction of autoimmune encephalomyelitis. J. Immunol. 159:3100-3103.

13. Suvannavejh, G.C., Dal Canto, M.C., Matis, L.A., and Miller, S.D. 2000 Fas-mediated apoptosis in clinical remissions of relapsing experimental autoimmune encephalomyelitis. J. Clin. Invest. 105:223-231.

14. Boise, L.H., et al. 1995. CD28 costimulation can promote T cell survival by enhancing the expression of Bcl-XL. Immunity. 3:87-98.

15. Noel, P.J., Boise, L.H., Green, J.M., and Thompson, C.B. 1996. CD28 costimulation prevents cell death during primary $\mathrm{T}$ cell activation. $J$. Immunol. 157:636-642.

16. Sperling, A.I., et al. 1996. CD28/B7 interactions deliver a unique signal to naive $\mathrm{T}$ cells that regulates cell survival but not early proliferation. $J$. Immunol. 157:3909-3917.

17. Wells, A.D., et al. 1999. Requirement for T-cell apoptosis in the induction of peripheral transplantation tolerance. Nat. Med. 5:1303-1307.

18. Issazadeh, S., Navikas, V., Schaub, M., Sayegh, M.H., and Khoury, S.J. 1998. Kinetics of expression of costimulatory molecules and their ligands in murine relapsing experimental autoimmune encephalomyelitis in vivo. J. Immunol. 161:1104-1112.

19. Issazadeh, S., Zhang, M., Sayegh, M.H., and Khoury, S.J. 1999. Acquired thymic tolerance: role of CTLA4 in the initiation and maintenance of tolerance in a clinically relevant autoimmune disease model. J. Immunol. 162:761-765

20. Slavin, A., et al. 1998. Induction of a multiple sclerosis-like disease in mice with an immunodominant epitope of myelin oligodendrocyte glycoprotein. Autoimmunity. 28:109-120.

21. Deibler, G.E., Martenson, R.E., and Kies, M.W. 1972. Large scale preparation of myelin basic protein from central nervous tissue of several mammalian species. Prep. Biochem. 2:139-165.

22. Schmid, I., Uittenbogaart, C.H., Keld, B., and Giorgi, J.V. 1994. A rapid method for measuring apoptosis and dual-color immunofluorescence by single laser flow cytometry. J. Immunol. Methods. 170:145-157.

23. Tabi, Z., McCombe, P.A., and Pender, M.P. 1995. Antigen-specific downregulation of myelin basic protein-reactive $\mathrm{T}$ cells during spontaneous recovery from experimental autoimmune encephalomyelitis: further evidence of apoptotic deletion of autoreactive $T$ cells in the central nervous system. Int. Immunol. 7:967-973.

24. White, C.A., McCombe, P.A., and Pender, M.P. 1998. The roles of Fas, Fas ligand and $\mathrm{Bcl}-2$ in $\mathrm{T}$ cell apoptosis in the central nervous system in experimental autoimmune encephalomyelitis. J. Neuroimmunol. 82:47-55.

25. Chen, Y., Hancock, W.W., Marks, R., Gonnella, P., and Weiner, H.L. 1998. Mechanisms of recovery from experimental autoimmune encephalomyelitis: $\mathrm{T}$ cell deletion and immune deviation in myelin basic protein T cell receptor transgenic mice. J. Neuroimmunol. 82:149-159.

26. Bonetti, B., Pohl, J., Gao, Y.L., and Raine, C.S. 1997. Cell death during autoimmune demyelination: effector but not target cells are eliminated by apoptosis. J. Immunol. 159:5733-5741. 